\title{
HERRAMIENTA DE SEGURIDAD OPERACIONAL EN FRANJA DE PISTA CON MODELOS PROBABILÍSTICOS DE EXCURSIONES DE PISTA
}

\author{
Federico Pinto ${ }^{1, *}$, Alejandro di Bernardi ${ }^{1,+}$, Mattias Coppa ${ }^{1, \neq}$, \\ Santiago Pezzotti ${ }^{1,}$, Sergio Pitrelli ${ }^{1}$
}

\begin{abstract}
${ }^{1}$ Grupo de Transporte Aéreo, UIDET GTA GIAI (Unidad de Investigación, Desarrollo, Extensión y Transferencia Grupo de Transporte Aéreo/Grupo de Ingeniería Aplicada a la Industria), Dto. Aeronáutica, Facultad de Ingeniería, Universidad Nacional de La Plata, 1900 Buenos Aires, Argentina
\end{abstract}

Recibido: $15 /$ junio/2019

Aceptado: 27/agosto/2019

DOI: https://doi.org/10.5377/ce.v12i2.10286

\section{RESUMEN}

En el presente trabajo se realiza el desarrollo de una herramienta de análisis de seguridad operacional en franja de pista mediante modelos probabilísticos de accidentes por excursiones de pista. Se presenta una herramienta preliminar que permite caracterizar y evaluar el riesgo operacional presente en franjas de pista y zonas asociadas en aeropuertos con operaciones aerocomerciales. Las conclusiones y recomendaciones obtenidas como resultado permiten lograr un aporte significativo para una mejora en la seguridad operacional. Adicionalmente se valida el desarrollo mediante la aplicación de la herramienta desarrollada en un determinado aeropuerto específico de la región SAM (South America) de OACI. Mediante la información bibliográfica recopilada, se llevó a cabo el análisis de las metodologías existentes asociadas a modelos de riesgo. Se analizaron las problemáticas más habituales en franjas de pista de aeropuertos con tráfico aerocomercial de la región SAM. Con la información obtenida y los criterios adoptados se procedió a identificar los principales factores de riesgo en las franjas de pista y zonas asociadas. Mediante la caracterización de los accidentes ocurridos se determinó la severidad de cada peligro existente, lo cual sirve como base para el desarrollo de la herramienta capaz de identificar el riesgo preliminar y evaluarlo. Se identificaron los aeropuertos más significativos respecto a operaciones en la región; y luego se aplicó la herramienta desarrollada en uno de ellos, para validar la misma.

Palabras clave: Seguridad operacional, franja de pista, aeropuerto, probabilidad, excursiones de pista.

\section{ABSTRACT}

This paper is about the development of a tool for operational safety analysis in the runway strip, using probabilistic models of runway excursion accidents. A preliminary tool is presented to characterize and evaluate the operational risk present in runway strips and associated areas in airports with commercial

\footnotetext{
*federico.matias.pinto@gmail.com

†aledibernardi@hotmail.com

‡matias.coppa@gmail.com

$\S_{\text {santiago.pezzotti@ing.unlp.edu.ar }}$
} 
air operations. The conclusions and recommendations obtained as a result allow achieving a significant contribution to an improvement in operational safety. In addition, the development is validated through the application of the tool developed in a specific airport in the ICAO SAM region.

Through the bibliographic information collected, the analysis of the existing methodologies associated with risk models was carried out. The most common problems were analyzed in runway strips of airports with commercial air traffic of the SAM region. With the information obtained and the criteria adopted it proceeded to identify the main risk factors in the runway strips and associated areas. Through the characterization of the accidents that occurred, the severity of each existing risk was determined, which serves as a basis for the development of the tool able to identify the preliminary risk and evaluate it. The most significant airports in the region were identified with respect to operations, and then the tool developed was applied in one of them was to validate it.

Keywords: Operational safety, runway strip, airport, propability, runway excursion.

\section{Introducción}

La herramienta desarrollada mediante modelos probabilísticos busca evaluar de manera integral situaciones de conflicto que son habituales en los aeropuertos de la región, y que ante una excursión de pista pueden ser un agravante. El usuario de la herramienta podrá de manera rápida, tener una noción preliminar del nivel de seguridad operacional respecto a un peligro identificado.

\subsection{Contexto aéreo mundial}

En la actualidad, el transporte aéreo es considerado como uno de los medios de transporte más seguros y confiables del mundo, sin embargo, no siempre ha sido así. Los avances en materia de seguridad operacional, entre los más relevantes, fueron impulsados por numerosas investigaciones realizadas sobre los diferentes accidentes ocurridos a lo largo de la historia. Dichas investigaciones permitieron detectar, identificar y cuantificar las causas de los accidentes, lo cual posibilitó el desarrollo de las medidas necesarias para mejorar la seguridad y confiabilidad del sistema aeronáutico.

De acuerdo con la Organización de la Aviación Civil Internacional (OACI, 2015b), la cantidad de vuelos comerciales en todo el mundo para el año 2016 fue de 34.5 millones, los cuales representan un crecimiento mayor al $20 \%$ en el transcurso de esta última década.

Teniendo en cuentas estas cifras y sumando información emitida en reportes anuales respecto a accidentes tanto de OACI como de IATA, se puede concluir que las estadísticas correspondientes a accidentes están decreciendo en el número total de accidentes año tras año, a pesar del incremento de la actividad aerocomercial. A continuación, se puede observar en la figura 1 el registro de accidentes de OACI (OACI, 2011, 2015a, 2013b) para el período 2005-2015, en el cual se muestra la relación entre el total de accidentes, cuántos de ellos han tenido consecuencias fatales, y la cantidad de fatalidades producidas por estos accidentes. 


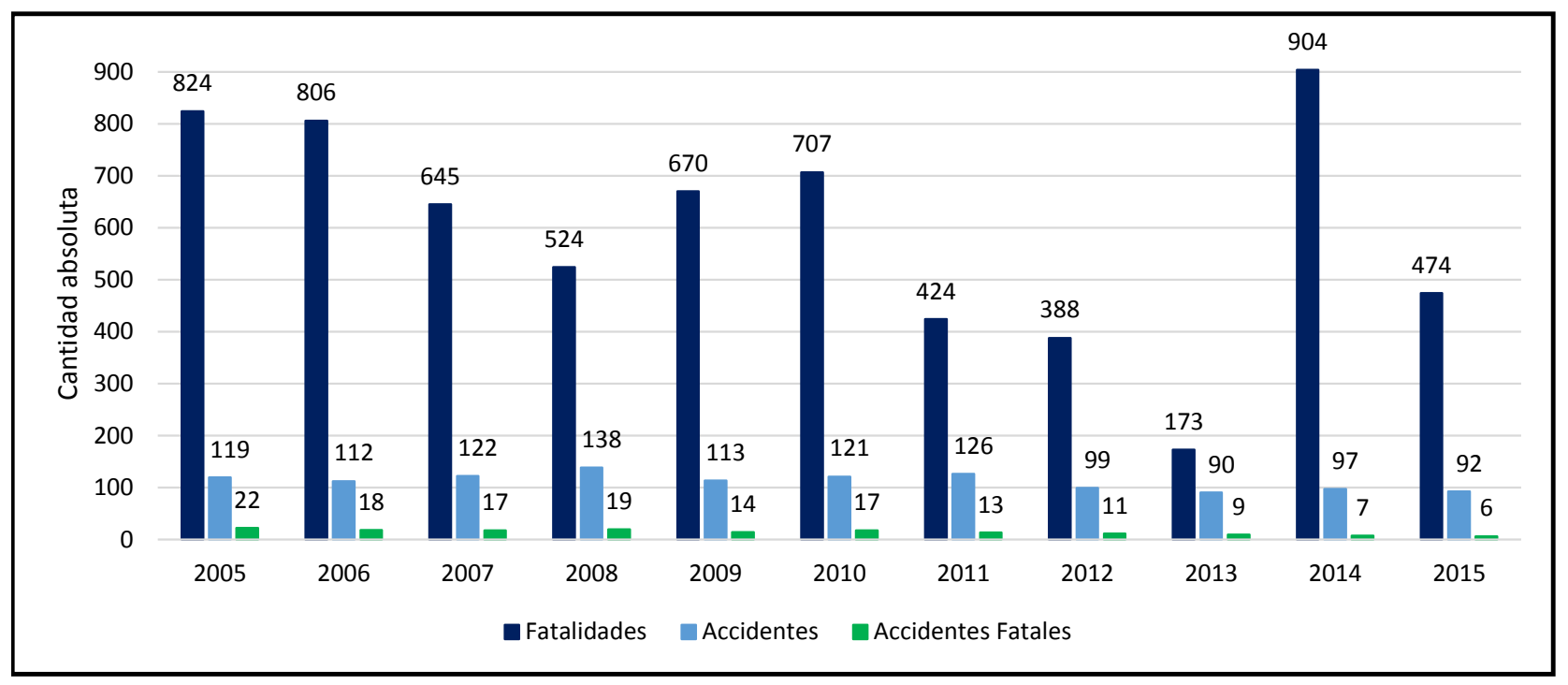

Figura 1: Registro de accidentes y fatalidades (2005-2015). Fuente: OACI (2016b)

Para tener una medición más representativa respecto de los accidentes, OACI elabora un índice de accidentes el cual se relaciona directamente con la cantidad de vuelos. A continuación, en la figura 2 se puede ver el resultado del índice de accidentes de manera anual para el período 2005-2015, en la cual se puede ver que dicho índice ha ido decreciendo, hasta alcanzar un valor de 2.8 accidentes por cada millón de vuelos para el año 2015, el cual es el registro más bajo junto con el del año 2013.

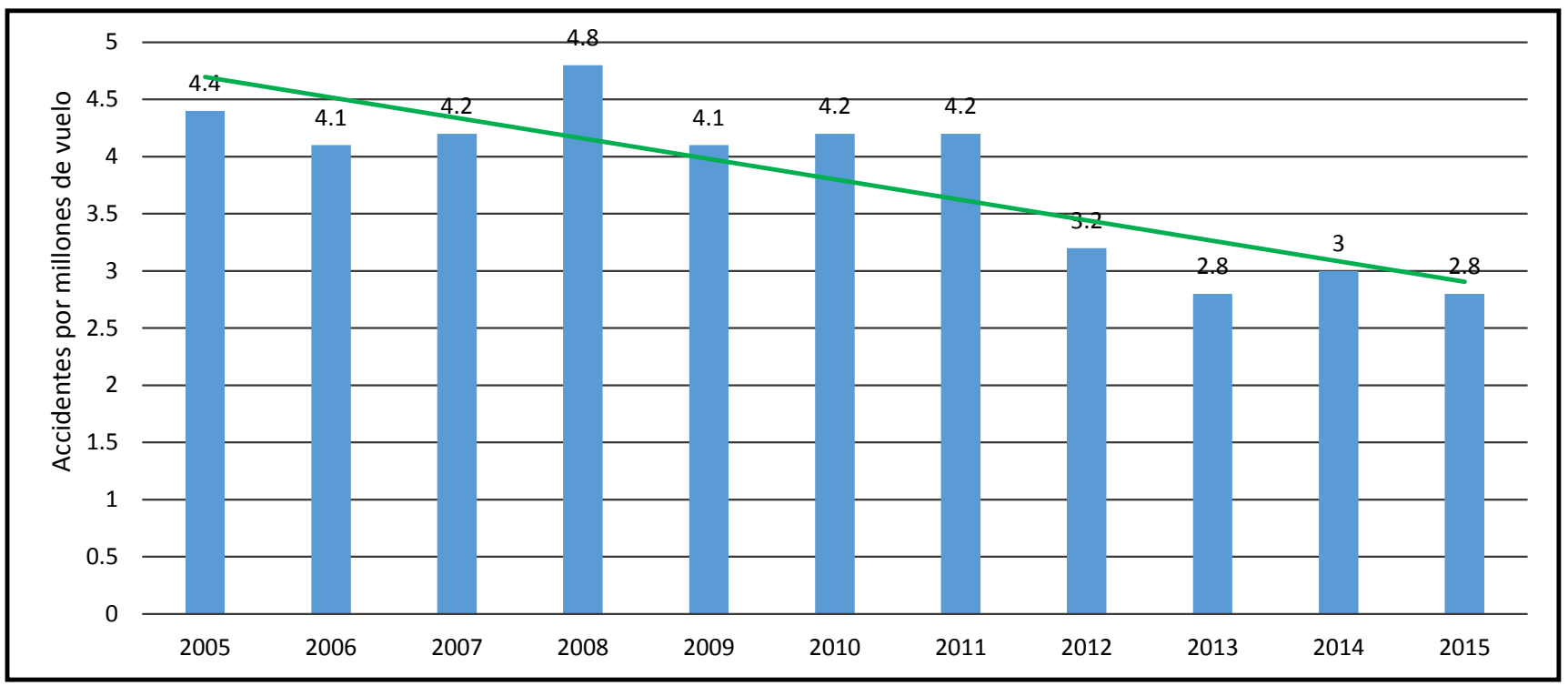

Figura 2: Índice de accidentes por cada millón de vuelos en el mundo. Fuente: OACI (2016b)

Analizando más en detalle los accidentes aéreos, se tiene que los mismos son categorizados en distintos tipos de sucesos, de los cuales para el interés del presente estudio, se analizan las excursiones de pista (RE por sus siglas en inglés Runway Excursion). Como podemos ver en la figura 3 y la figura 4, estos accidentes son los más frecuentes en los últimos períodos analizados. 


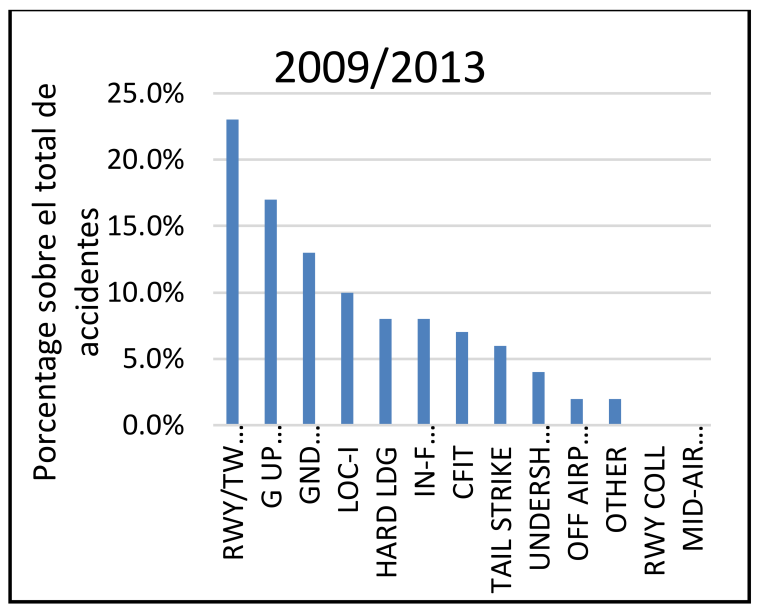

Figura 3

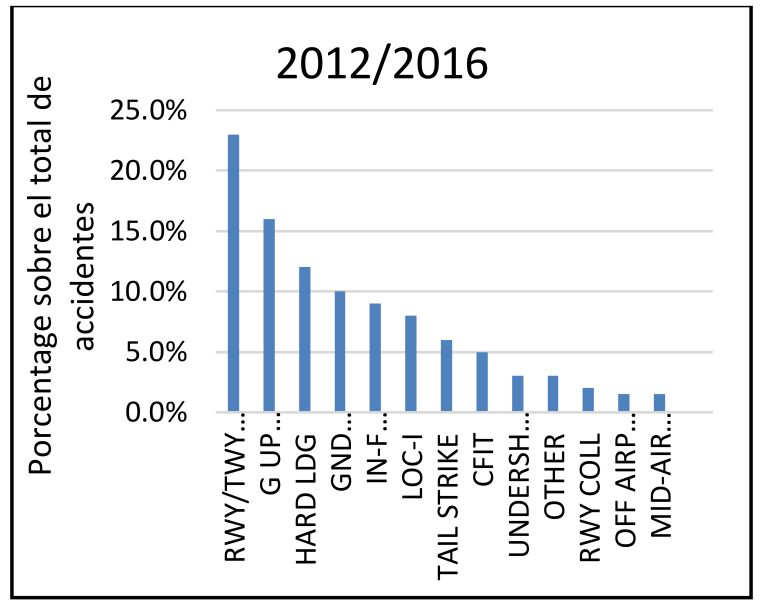

Figura 4

Accidentes por categoría para períodos 2009/2013 y 2012/2016. Fuente: (IATA, 2013, 2017).

\subsection{Excursiones de pista}

Una excursión de pista, RE es cualquier evento en el que una aeronave se desvía o rebasa la superficie de la pista ocurriendo durante la fase de despegue o aterrizaje. Se distingue entre dos diferentes tipos de excursiones de pista, veer-off y overrun, las cuales se categorizan según la posición final de la aeronave $\mathrm{o}$, dicho de otra manera, en qué sector de la pista se produzca la excursión.

Para el período 2004-2009 ocurrieron un total de 164 accidentes que fueron categorizadas como Excursiones de Pista (27\%) (IATA, 2010). De manera similar, para el período de 2009 - 2013 se tuvieron 98 RE (22.7\%) (IATA, 2013) y para el período $2012-2016,82$ (21.8\%) (IATA, 2017) fueron categorizadas como RE. Por lo tanto, estas estadísticas evidencian que, a nivel mundial, los esfuerzos por minimizar estos accidentes vienen proporcionando resultados favorables, pero con una tendencia mínima, por lo que se debe atacar aun con más fuerza esta problemática. En la tabla 1 se observa un análisis comparativo de las excursiones de pista comparadas con un total de accidentes a lo largo de los años.

Tabla 1: Contribución de excursiones de pista (RE) sobre el total de accidentes. Fuente: (IATA, 2010, 2013, 2017)

\begin{tabular}{lccc}
\hline Observación & $\mathbf{2 0 0 4 - 2 0 0 9}$ & $\mathbf{2 0 0 9} \mathbf{- 2 0 1 3}$ & $\mathbf{2 0 1 2}$ - 2016 \\
\hline Total de accidentes & 594 & 432 & 375 \\
\hline Cantidad de RE & 164 & 98 & 82 \\
\hline$\%$ RE & $27 \%$ & $23 \%$ & $22 \%$
\end{tabular}

En un aeropuerto existen diferentes áreas de seguridad, destinadas a reducir el riesgo de daños a las aeronaves que se salgan de la pista, por lo que en este tipo de accidente son de suma importancia. Una de ellas es la RESA (área de seguridad de extremo de pista) y la otra es la franja de pista. Según los reportes de IATA ya citados anteriormente, los overruns son más frecuentes que los veer-off, por lo que se debe prestar especial atención en las aéreas de RESA ubicadas sobre las prolongaciones de la pista. Se tiene un promedio de 3.6 overruns por mes en el mundo. 


\subsection{Estudio de seguridad operacional (ESO)}

Que un aeropuerto tenga inconvenientes a la hora de certificar sus instalaciones, no quiere decir que no pueda operar, para estos casos es que existen los llamados Estudios de Seguridad Operacional, más conocidos también de manera más abierta como como Estudios Aeronáuticos. El objetivo de los mismos, es demostrar mediante trabajos técnicos por parte del gestor aeroportuario, que es posible alcanzar un nivel de seguridad no menor al que se alcanzaría a través de un cumplimiento estricto de la especificación. Las principales no conformidades en los aeródromos disparadores de estudios de seguridad operacional suelen estar asociadas a la RESA, Franja de pista, Anchos de pistas y calles de rodaje, Separaciones entre pistas y calles de rodaje, Obstáculos (árboles, edificios, etc.).

Para poder definir el riesgo asociado a un peligro, es necesario calcular la probabilidad y la potencial severidad del mismo. La severidad hace referencia a cuan grave podría llegar a ser la situación, ante un eventual accidente, mientras que la probabilidad hace referencia a qué posibilidad existe no solo de que ocurra un accidente sino de que, en ese eventual accidente, la aeronave termine viéndose afectada por el peligro identificado.

Todos y cada uno de los peligros deben tener asociado algún riesgo que determina la tolerabilidad del peligro, la cual varía según las autoridades de cada país en base a consideraciones propias. Para poder definir correctamente dicha tolerabilidad de cada uno de los riesgos, lo que se hace es situar los mismos dentro de lo que se conoce como Matriz de Riesgo (ver figura 5), la cual es el producto final de un estudio aeronáutico.

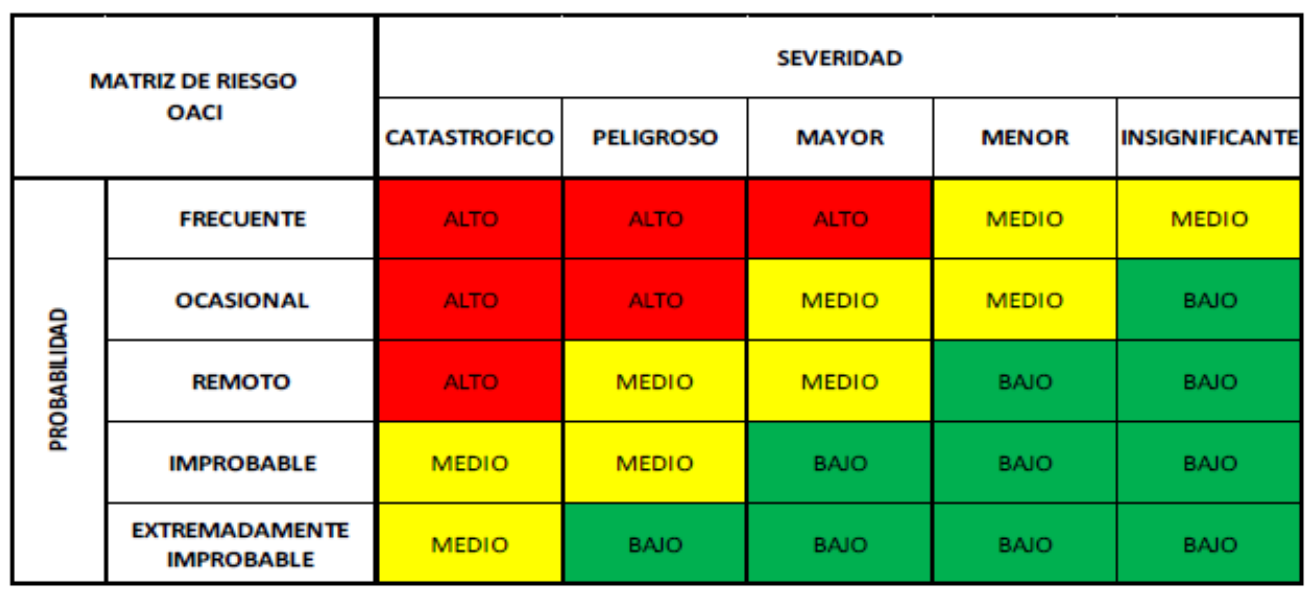

Figura 5: Matriz de riesgo según OACI. Fuente: OACI (2013a)

\section{Metodología}

La metodología que se aplicó para llevar a cabo este trabajo es la que se detalla a continuación. Primero se hace un análisis de los aeropuertos de la región SAM y se selecciona los aeropuertos a estudiar. Luego se hace la identificación de peligros, para lo cual se crea un modelo gráfico el cual representa las áreas bajo análisis y se identifican de peligros existentes en cada aeropuerto. A continuación, se desarrolla la herramienta de seguridad operacional, para la cual se crea un modelo para determinar la probabilidad del suceso y se crea un modelo para determinar la severidad del peligro. Por último, se lleva a cabo un caso de aplicación, en el cual se visualizan los resultados de la herramienta mediante una matriz de riesgo. 


\subsection{Identificación de peligros}

El análisis que se llevó a cabo para este trabajo, fue realizado en la región Sud Americana de OACI (SAM), la cual está constituida por 13 países, Argentina, Bolivia, Brasil, Chile, Colombia, Ecuador, Guayana Francesa, Guyana, Paraguay, Perú, Surinam, Uruguay y Venezuela seleccionando los dos principales aeropuertos respecto a la cantidad de operaciones para cada uno de los países de la región, con algunas excepciones particulares.

El modelo gráfico desarrollado para el análisis de la identificación de peligros, distingue de manera rápida zonas de seguridad de extremo de pista, tanto obligatorias como recomendadas según OACI (OACI, 2005, 2016a); también distingue diferentes sectores de la franja de pista. Para la franja de pista se realizó una discretización que permite no solo evidenciar rápidamente las zonas de interés, sino que también se ajusta de manera adecuada a los modelos probabilísticos correspondientes a veeroff y overrun que se explican más adelante. A continuación, en las figuras 6 y 7 se pueden ver dos imágenes que muestran la visualización del modelo con las imágenes satelitales estudiadas.

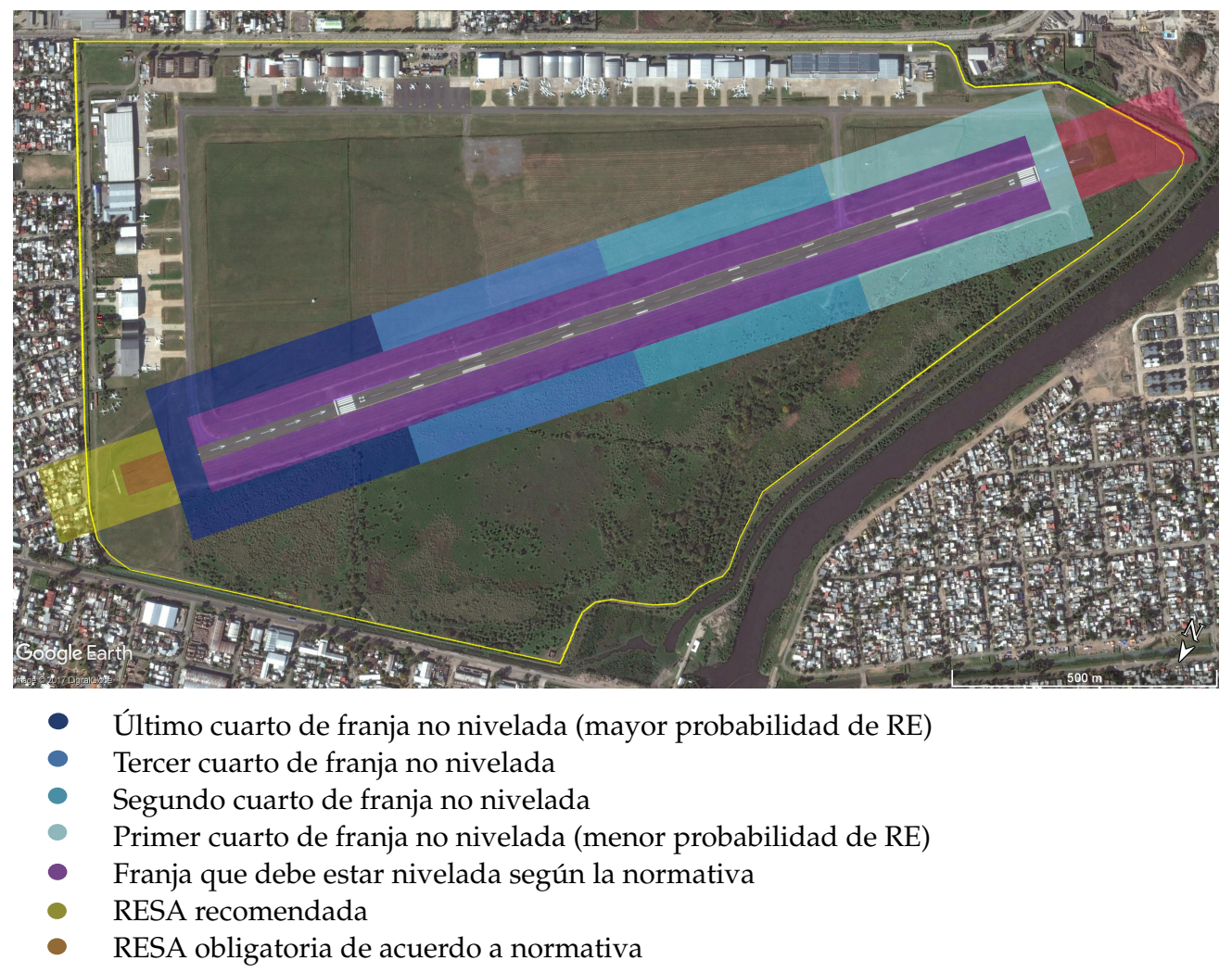

Figura 6: Visualización del modelo gráfico con imágenes satelitales. 


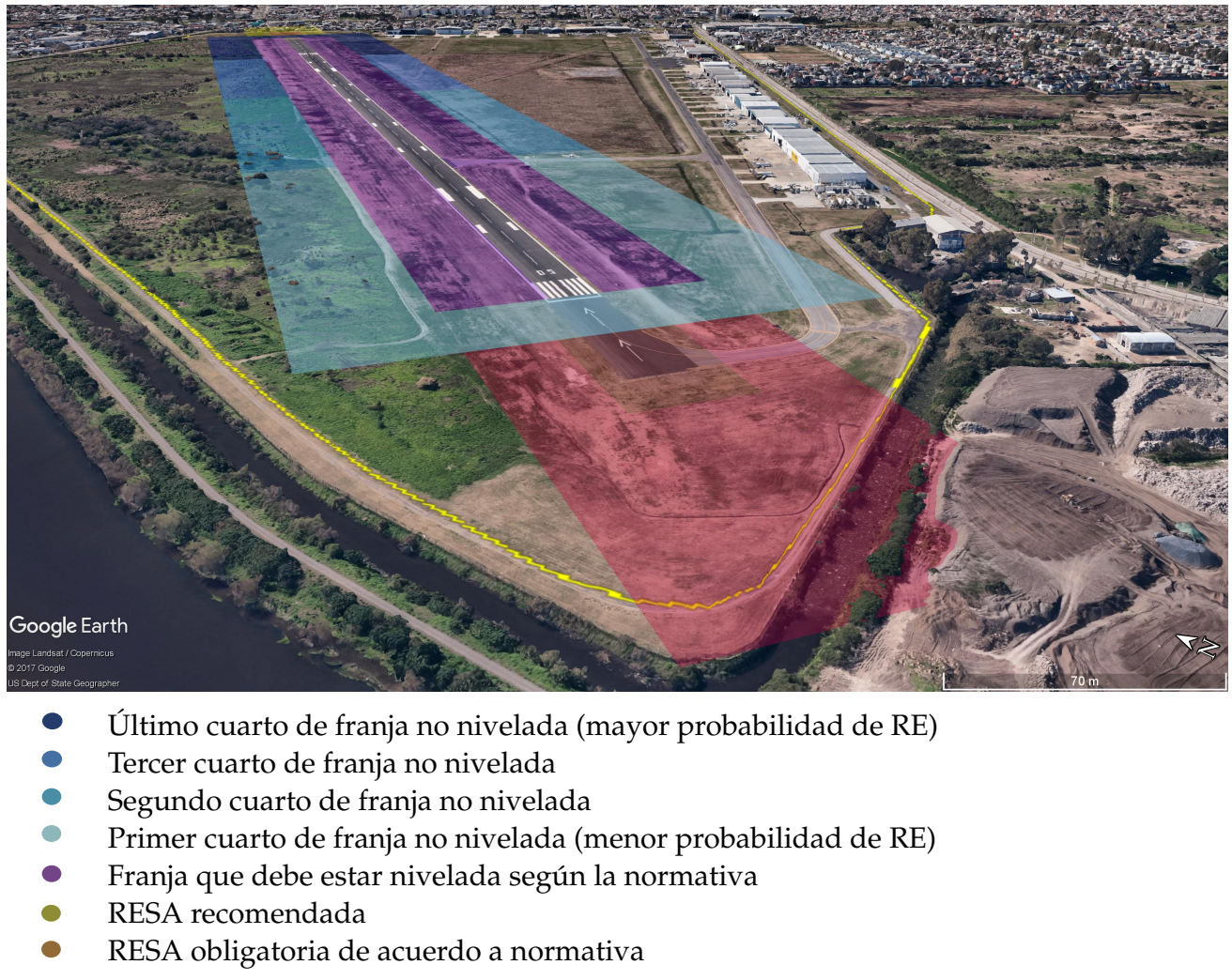

Figura 7: Visualización del modelo gráfico con imágenes satelitales.

Luego de visualizar el modelo gráfico, con la metodología de análisis mencionada, los peligros que se identificaron son los que se detallan a continuación: cuerpos de agua, cercos perimetrales, arboles, deflectores de flujo, aeronaves fuera de servicio, playas de estacionamiento, autopistas o rutas, plataformas de helipuerto, plataformas de aeronaves, discontinuidades abruptas de terreno, edificaciones de sistemas de apoyo, edificaciones urbanas, edificaciones aeroportuarias, edificios terminales y plantas de combustible.

\subsection{Determinación probabilística de los sucesos}

Cada peligro identificado en un cuadrante determinado, puede tener una alta probabilidad de ser impactado durante una excursión de pista en un sentido de operación, pero puede tener una muy baja probabilidad en el sentido opuesto de operación. Mientras que por otro lado la probabilidad de ocurrencia sería la misma independientemente del peligro identificado y permite clasificar los eventos de acuerdo a la frecuencia con la que ocurren los mismos en base a datos de antecedentes. Por lo tanto, como deben ocurrir ambos sucesos simultáneamente la probabilidad final es el producto de las otras probabilidades mencionadas. Es decir, que no solo tiene que suceder una excursión de pista, sino que también la aeronave debería terminar en un sector determinado donde se ubicaría el peligro. Por lo tanto, el modelo para hallar la probabilidad final de un peligro específico es el siguiente:

$$
P_{\text {total }}=P_{\text {ocurrencia }} \times P_{\text {localización }}
$$

Para el desarrollo de la herramienta en cuestión, se utilizó un modelo de cálculo el cual distingue la cantidad de excursiones de pista diferenciadas entre veeroff y overruns, en base a la cantidad de accidentes 
y a la cantidad de operaciones de toda la región bajo análisis. Por lo tanto, el modelo para el cálculo de la probabilidad de ocurrencia es el que se muestra a continuación:

$$
\begin{aligned}
& P_{\text {ocurrencia OR }}=\frac{\text { accidentes }}{\text { operaciones }} \times \frac{\text { excursiones de pista }}{\text { accidentes }} \times \frac{\text { overrun }}{\text { excursiones de pista }} \\
& P_{\text {ocurrencia VO }}=\frac{\text { accidentes }}{\text { operaciones }} \times \frac{\text { excursiones de pista }}{\text { accidentes }} \times \frac{\text { veeroff }}{\text { excursiones de pista }}
\end{aligned}
$$

Respecto a la probabilidad de localización, se debe mencionar que es de suma importancia poder describir mediante estadísticas la posición final aproximada de cada aeronave. Para ello se utilizan modelos desarrollados por la ACRP (ACRP, 2014), capaces de distinguir entre veeroff y overrun.

Cada cuadrante de la discretización establecida tiene asociada una probabilidad de localización correspondiente, la cual a su vez está conformada por el producto de una probabilidad respecto al eje longitudinal de pista $(x)$ y respecto al eje transversal de pista $(y)$, los cuales también dependen del tipo de suceso analizado.

$$
P_{\text {localización }}=P_{x} \times P_{y}
$$

A modo de resumen de lo recientemente descrito, a continuación se presenta en las figuras 8 y 9 el sistema de coordenadas " $x$ " e " $y$ " para cada modelo dependiendo el suceso analizado.

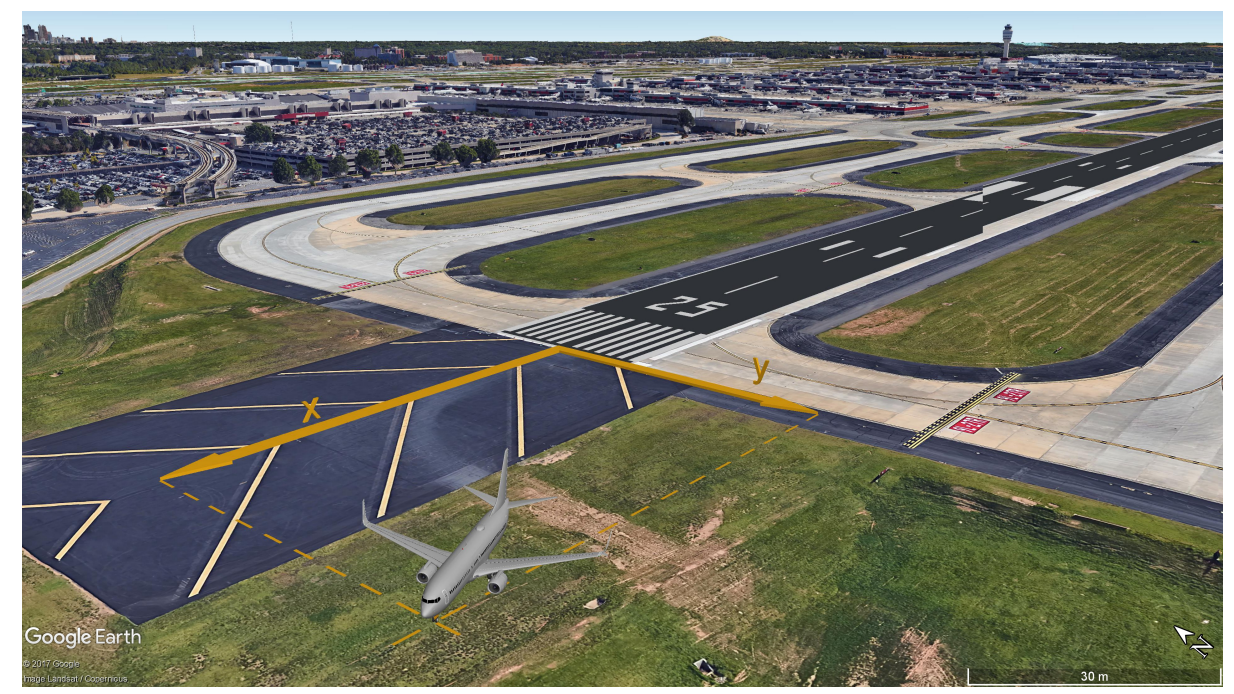

Figura 8: Sistema de coordenadas para modelo de Overrun. 


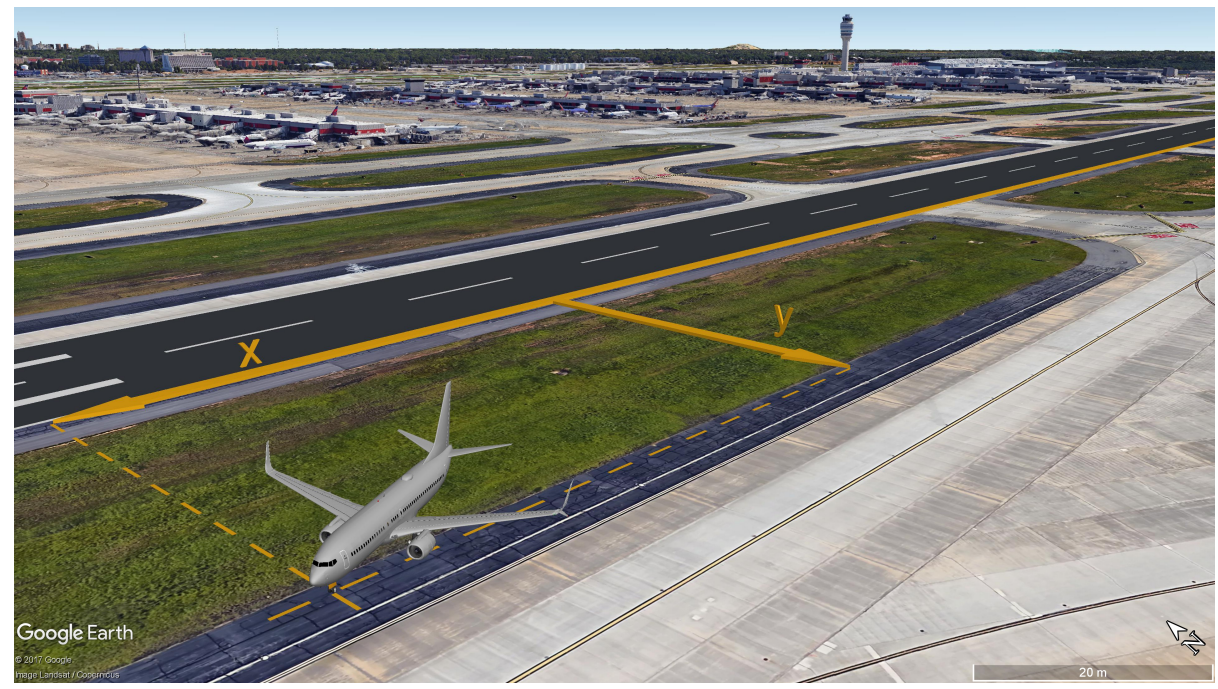

Figura 9: Sistema de coordenadas para modelo de Veer-Off.

Luego del análisis estadístico y de ajustar los modelos probabilísticos a la discretización utilizada, se desarrolló la probabilidad de localización para cada uno de los cuadrantes, la cual se puede ver en la figura 10.

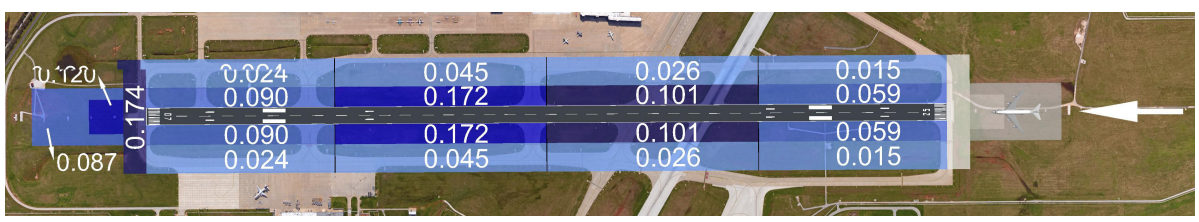

Figura 10: Visualización de probabilidad de localización.

Combinando la probabilidad de localización obtenida recientemente, con las probabilidades de ocurrencia para cada una de las diferentes operaciones, se tiene la probabilidad final para cada uno de los cuadrantes que se muestra a continuación en la figura 11.

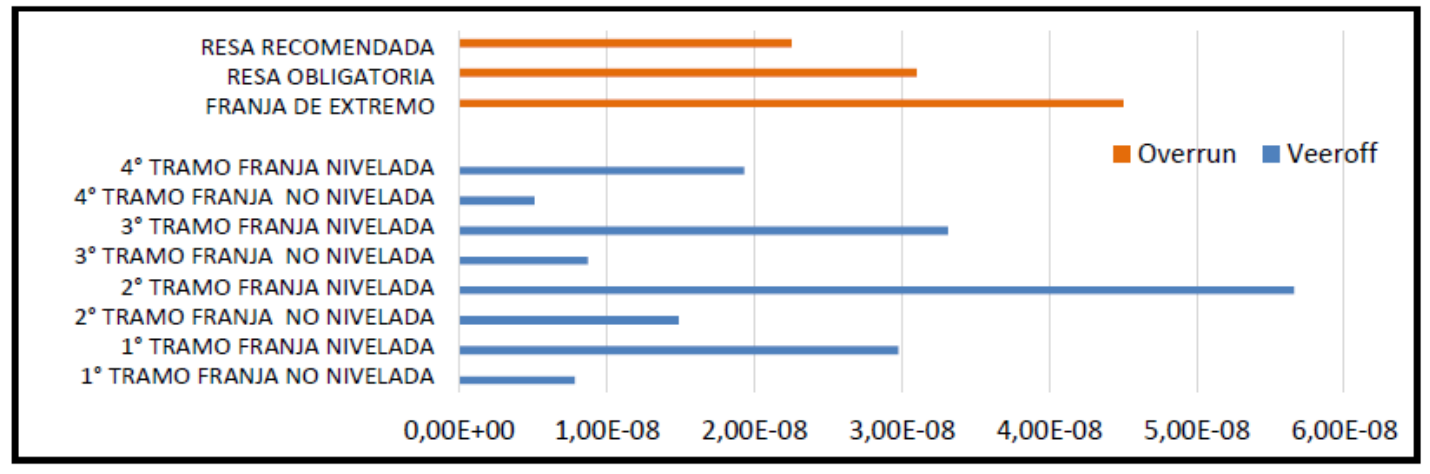

Figura 11: Probabilidad final por cuadrantes por veeroff y overrun. 


\subsection{Ponderación de severidad para peligros identificados}

En función de poder desarrollar la herramienta de análisis de seguridad operacional, se debe estimar la severidad asociada a un peligro, y para ello es necesario conocer qué factores relevantes intervienen, los cuales pueden actuar como agravantes o atenuantes. Estos factores relevantes tenidos en cuenta a la hora de evaluar la severidad son los siguientes: tipo de peligro, ubicación del peligro y la aeronave involucrada (ver figura 12).

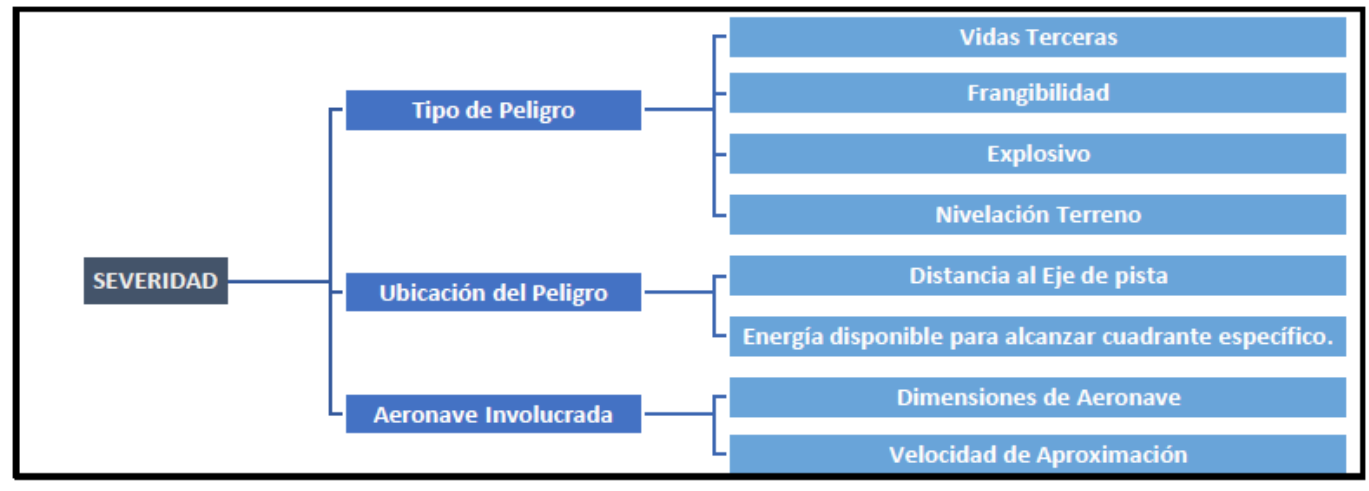

Figura 12: Factores relevantes a la severidad de un accidente.

Respecto al primer factor relevante, tipo de peligro identificado, la herramienta será capaz de poder evidenciar ciertas características físicas del peligro. Por lo tanto, se hizo una caracterización que sirvió para ponderar y determinar una prelación entre los peligros considerando diferentes criterios, como el nivel de frangibilidad, si se corresponde a desnivelaciones en el terreno, si tienen características explosivas y si suma vidas de terceros al accidente.

Respecto al segundo factor, la ubicación de los peligros identificados, se definió un criterio de manera que cada cuadrante de la discretización planteada tenga asignado un valor ponderado de severidad. Para que los diferentes cuadrantes tengan asociado un nivel de severidad, lo que se llevó a cabo es un análisis de energía asociada a una aeronave durante una excursión de pista, dentro de diferentes escenarios hipotéticos. En otras palabras, considerando diferentes aeronaves y diferentes puntos de toma de contacto en el aterrizaje, se evaluó la capacidad de disminuir la energía acumulada de la aeronave por medio de una desaceleración lineal.

Respecto a la aeronave involucrada, se caracterizó la composición de una flota que sea lo más representativa posible de la región, y para ello se consideraron un total de 83 aeronaves. Las aeronaves fueron categorizadas en tres diferentes tipos según sus dimensiones, diferenciándolas directamente por su tipo de fuselaje, y por otro lado, se caracterizaron en cuatro niveles según la velocidad de aproximación. 


\section{Resultados}

Sobre un total de 26 aeropuertos analizados, y considerando los diferentes sentidos de operaciones para sus respectivas pistas, lo que indica un total de 72 pistas operativas, se identificaron un total de 848 peligros dentro de las zonas bajo análisis. En la figura 13 se puede observar la distribución de la cantidad de peligros identificados, relacionados con la cantidad de operaciones anuales para cada aeropuerto identificado con su código IATA.

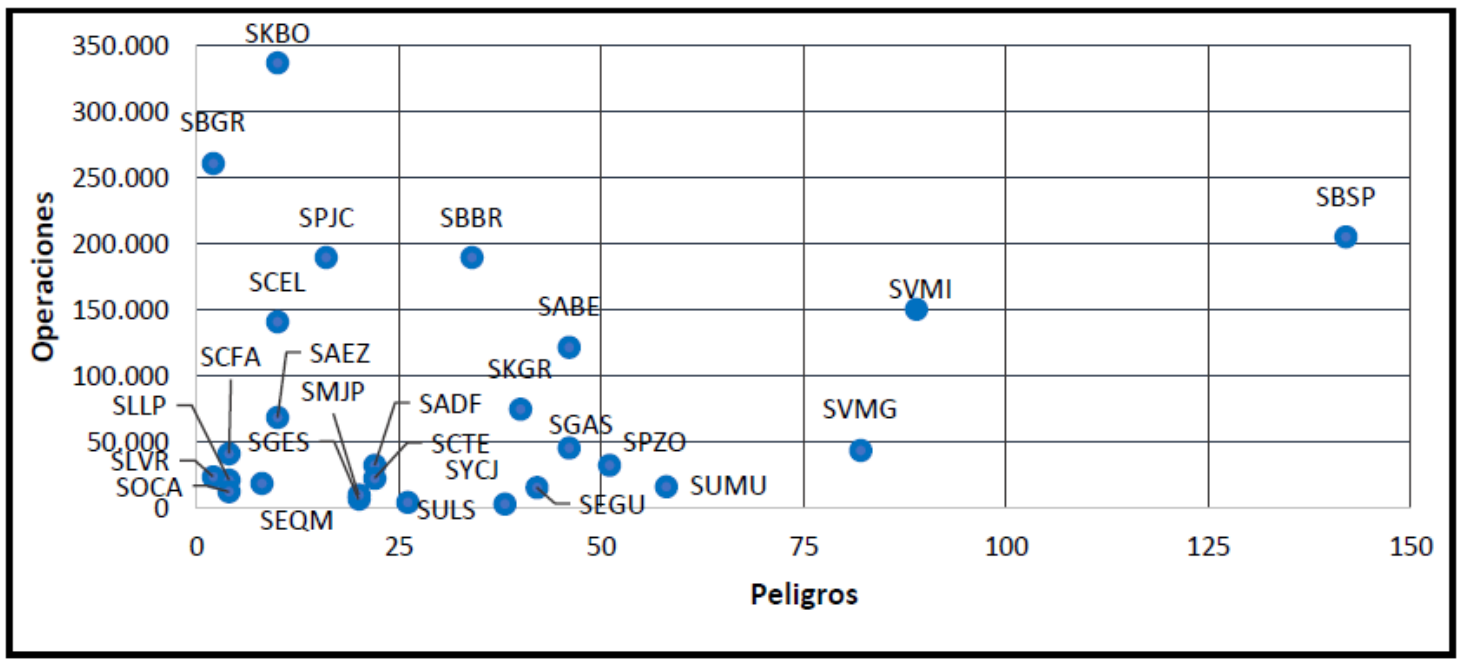

Figura 13: Cantidad de peligros vs cantidad de operaciones por aeropuerto.

Además, sobre el total de estos peligros identificados, también se puede decir que más del $75 \%$ de los mismos fueron identificados dentro de cinco tipos de peligros, siendo estos un $34 \%$ "cercos perimetrales", el 26\% "arboles", 9\% "discontinuidades de terreno", $7 \%$ "autopistas o rutas" y un $6 \%$ en "edificaciones de sistemas de apoyo". Finalmente, la herramienta desarrollada, combina de manera sencilla, rápida y eficaz, mediante una interfaz amigable en Excel, cada uno de los modelos, análisis y criterios utilizados en el desarrollo del presente trabajo. La interfaz de la herramienta permite al usuario poder seleccionar dentro de tres menús desplegables que le permiten clasificar el riesgo identificado que el usuario debe completar para que la herramienta pueda asignar un nivel de probabilidad y severidad al peligro. En la figura 14 se presenta el esquema de datos de entrada necesarios para la herramienta.

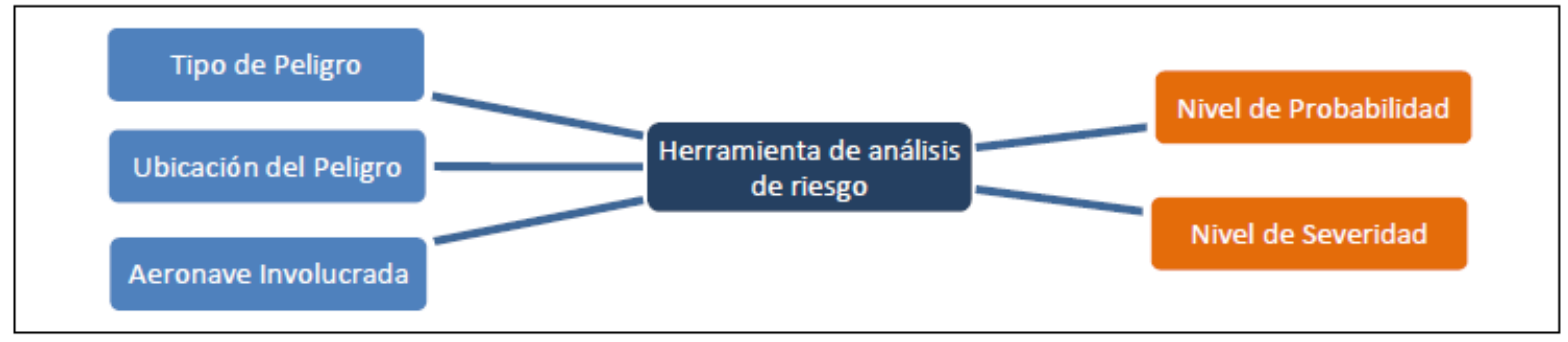

Figura 14: Esquema de datos de entrada necesarios para la herramienta.

Lo primero que debe hacer el usuario, es seleccionar los tres casilleros blancos, los cuales son entradas obligatorias y se corresponden con los factores relevantes analizados. Hecho esto, la herramienta devuelve tanto una estimación de severidad como de probabilidad. 
A modo de ejemplo, la figura 15 muestra la interfaz de la herramienta con los resultados concretos para el caso de una discontinuidad abrupta de terreno, ubicada en el $2^{\circ}$ tramo de franja nivelada, operando con un Boeing 787; donde la herramienta determina una severidad catastrófica y una probabilidad extremadamente remota.

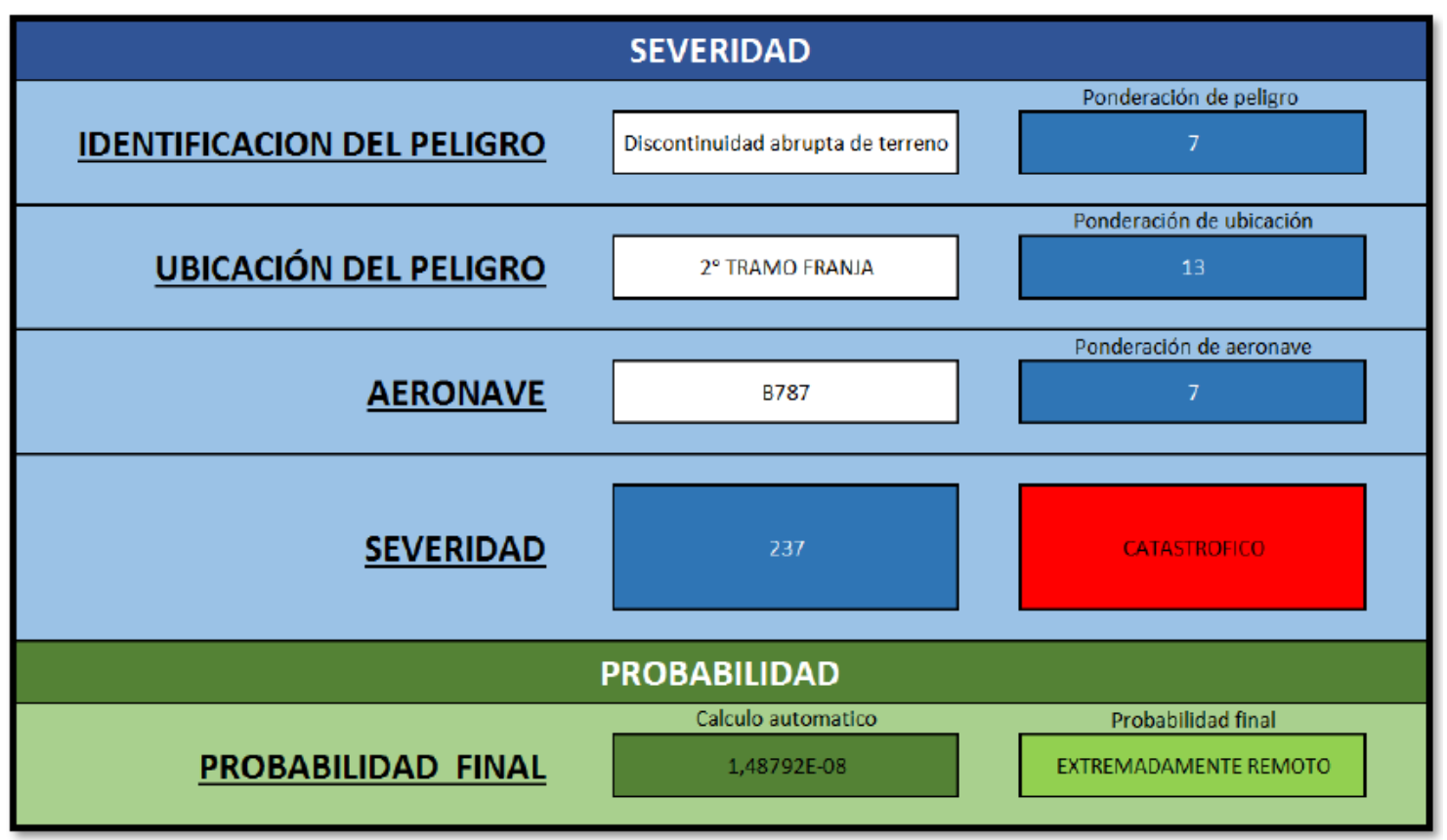

Figura 15: Ejemplos de utilización de herramienta.

Es importante destacar que, la herramienta es capaz de determinar la severidad y la probabilidad del peligro identificado, pero no analiza el riesgo del mismo dado a la diversidad que tiene cada país respecto a la tolerabilidad distinta a cada peligro como se explicó anteriormente.

\section{Conclusiones}

Como primera conclusión se puede decir que los resultados del análisis indican una evidente diferencia del nivel de riesgo asociado a un mismo peligro dependiendo del sentido de operación de pista. Esto es muy importante para el operador del aeropuerto, dado que se puede optar por buscar establecer medidas que busquen mitigar dicho riesgo mediante limitaciones operativas.

Por otro lado, es de suma importancia conocer los criterios de cada autoridad aeronáutica involucrada en un análisis de riesgo. Como se mencionó anteriormente, cada autoridad define su tolerabilidad al riesgo implicado en base a consideraciones propias, como así también del escenario de peligro y del tipo de actividad desarrollada. Por lo tanto, para los aeropuertos emplazados en diferentes países, un mismo peligro identificado, inclusive ubicado en un mismo cuadrante, puede requerir acciones inmediatas para bajar el nivel de riesgo, o bien puede significar un riesgo aceptable, simplemente por cambiar el nivel de tolerabilidad del riesgo, acorde al grupo de expertos interviniente y a la autoridad aeronáutica competente.

Sin embargo, se puede decir que para el $100 \%$ de los aeropuertos analizados se identificó al menos un peligro, ninguno está exento de peligros. Por lo tanto, esto pone en evidencia que, para la región completa de América del Sur, es necesario proyectar nuevos estudios aeronáuticos que permitan definir los riesgos 
y los niveles de tolerabilidad acordes, para que los aeropuertos puedan cumplir satisfactoriamente con los requerimientos y normativas vigentes.

Esta condición de los aeropuertos de la región, también pone en evidencia en cierta manera, la falta de planificación tanto con respecto del entorno urbano del aeropuerto como así también, respecto a la competencia contra su propia expansión, dado que muchos peligros identificados fueron propios del aeropuerto.

\section{Referencias}

ACRP (2014). Development of a runway veer-off location distribution risk assessment model and reporting template. Airport Cooperative Research Program (ACRP.

IATA (2010). Safety report 2004-2009. International Air Transport Association (IATA).

IATA (2013). Safety report 2009-2013. International Air Transport Association (IATA).

IATA (2017). Safety report 2012-2016. International Air Transport Association (IATA).

OACI (2005). Manual de diseño de aeródromos-volumen ii. Organización de Aviación Civil Internacional (OACI).

OACI (2011). Situación de la seguridad operacional de la aviación mundial. Organización de Aviación Civil Internacional (OACI).

OACI (2013a). Safety management manual(smm). Organización de Aviación Civil Internacional (OACI).

OACI (2013b). Situación de la seguridad operacional de la aviación mundial. Organización de Aviación Civil Internacional (OACI).

OACI (2015a). Informe de seguridad operacional. Organización de Aviación Civil Internacional (OACI).

OACI (2015b). Tablas relativas al mundo del transporte aéreo en 2015. Organización de Aviación Civil Internacional (OACI).

OACI (2016a). Anexo 14-aeródromos-volumen i. Organización de Aviación Civil Internacional (OACI).

OACI (2016b). Safety report. Organización de Aviación Civil Internacional (OACI). 